\title{
Determination of triple points in the phase diagram of praseodymium
}

\author{
Y. C. Zhao, ${ }^{*}$ F. Porsch, and W. B. Holzapfel \\ Fachbereich Physik, Universität-GH-Paderborn, D-33095 Paderborn, Germany
}

(Received 25 January 1995)

\begin{abstract}
The phase diagram for praseodymium under pressures up to $23 \mathrm{GPa}$ and temperatures up to $573 \mathrm{~K}$ is studied by in situ energy-dispersive x-ray diffraction with an externally heated diamond-anvil cell apparatus along the phase boundaries between $\operatorname{Pr}(\mathrm{II})$ ( $\mathrm{fcc}$ ), $\operatorname{Pr}$ (III) (distorted fcc), and $\operatorname{Pr}(\mathrm{IV})$ ( $\alpha$-uraniumtype structure). The triple point of these three phases is located around $21 \mathrm{GPa}$ and $537 \mathrm{~K}$, representing the end point of the apparently second-order phase transition between $\operatorname{Pr}(\mathrm{II})$ and $\operatorname{Pr}(\mathrm{III})$ at the transition to the high-pressure phase $\operatorname{Pr}(\mathrm{IV})$. Together with the compression data for $\operatorname{Pr}$ at ambient temperature, the present study extends the $P-T$ phase diagram up to the melting lines.
\end{abstract}

The structural systematics of the regular trivalent lanthanide $(\mathrm{Ln})$ metals (from $\mathrm{La}$ to $\mathrm{Lu}$ without $\mathrm{Ce}, \mathrm{Eu}$, and $\mathrm{Yb}$ ) have been studied near ambient pressures in a wide range of temperatures and under higher pressures in more restricted temperature ranges. ${ }^{1-9}$ After $\mathrm{Ce}, \mathrm{Pr}$ is the next Ln metal which shows strong $4 f$-electron contributions to its structural energy under pressure, manifested by the volume-collapse transition from $\operatorname{Pr}$ (III) (distorted fcc) to $\operatorname{Pr}(\mathrm{IV})$ ( $\alpha$-U-type structure) and by similarity in its resistivity behavior at the $\operatorname{Pr}($ III)- $\operatorname{Pr}($ IV) transition around $21 \mathrm{GPa}$ in comparison with the $\gamma-\alpha$ phase transition of $\mathrm{Ce},{ }^{6,9-11}$ which is generally related to $4 f$-electron delocalization. The boundaries in the $P-T$ phase diagram for $\operatorname{Pr}$ had been studied earlier by differential thermal analysis, ${ }^{12,13}$ x-ray diffraction, ${ }^{7}$ and at low temperatures by electrical resistivity measurements. Especially the results $^{9,14}$ on the $\operatorname{Pr}($ II)-Pr(III) and $\operatorname{Pr}($ III)-Pr(IV) boundaries gave hints for the possible existence of a new triple point. The primary intention of the present work was the search for this triple point, keeping in mind that the transition between $\operatorname{Pr}($ II) with its fcc structure and $\operatorname{Pr}($ III) with its distorted-fcc structure has been considered to be an apparently second-order phase transition, which could result in a special triple point at the strong first-order phase transitions ${ }^{5-8,15,16}$ to $\operatorname{Pr}(\mathrm{IV})$.

In addition, the unusually flat melting curve for $\operatorname{Pr}($ II) and some discrepancy with shock-wave data on melting under pressure raised the question as how these results could be reconciled with the existence of the highpressure phase $\operatorname{Pr}(\mathrm{IV})$ and with the indications of $4 f$ electron delocalization.

In the present measurements, pressure was generated with a diamond anvil cell (DAC). ${ }^{17,18}$ An external electrical resistivity heater was used to obtain homogenous temperatures in the sample up to $573 \mathrm{~K}$. The phases were identified by energy-dispersive $x$-ray diffraction (EDXD) with a tungsten fine-focus $x$-ray tube operated at $25 \mathrm{~mA}$ and $60 \mathrm{kV}$ and a conical collimator system with $2 \theta \approx 10^{\circ}$ placed in front of a large-area intrinsic Ge detector as described previously. ${ }^{19}$ The exact value of $2 \theta$ in each run was calibrated by EDXD spectra of gold powder. This system is especially suitable for highpressure-high-temperature EDXD studies due to the averaging over the whole diffraction ring, which improves the spectral quality for coarse-grained samples significantly. Mineral oil was used as the pressuretransmitting medium to prevent also the sample from oxidation during sample loading. This oil is obviously chemically inert to $\mathrm{Pr}$ up to at least $573 \mathrm{~K}$, because no spurious diffraction peaks could be detected. With the present experimental instrumentation, diffraction spectra recorded in less than $100 \mathrm{~s}$ have sufficient intensity to analyze the phase composition. The Pr sample material had been obtained from K. A. Gschneidner, Jr., with analysis of the major impurities in atomic ppm: 652 for oxygen, 23 for iron, and 17 for other rare metals.

To locate the phase boundaries, the x-ray-diffraction spectra were recorded either on heating and cooling at fixed load, starting with a defined pressure at room temperature, or by isothermal compression and decompression. In total, 69 EDXD spectra were recorded in these experiments. The temperatures were measured and controlled by a PID regulator with a calibrated ChromelAlumel thermocouple in contact to one diamond anvil. The pressures were measured at room temperature by the ruby fluorescence technique. ${ }^{20}$ At the higher temperatures the fluorescence signal of ruby $R$ lines became too weak for accurate pressure determinations. Since the diffraction spectra of the common pressure standards for high-temperature $\mathrm{x}$-ray measurements such as $\mathrm{Au}$ and $\mathrm{Pt}$ (Refs. 21 and 22) interfere seriously with the spectra of the sample, no extra internal pressure standards were used, but the pressures at elevated temperatures were evaluated directly from the measured volume of the $\mathrm{Pr}$ sample itself, taking into account that the thermal correction could be estimated with sufficient accuracy by the equation of state. From the $300-\mathrm{K}$ isotherm ${ }^{6}$ with a Debye temperature ${ }^{23} \Theta_{D}=151 \mathrm{~K}$, the approximation $\gamma / V=\gamma_{0} / V_{0}$, and a Slater-Grüneisen parameter $^{24}$ $\gamma_{S, 0}=0.83$, the maximum thermal pressure correction is only $0.5 \mathrm{GPa}$ (at $21 \mathrm{GPa}$ and $573 \mathrm{~K}$ ). Runs in the processes of heating and cooling at a fixed load indicated that the pressures evaluated by the above method were very close to the corresponding starting pressures at room temperature, with the maximum deviations of 0.3 $\mathrm{GPa}$ in the lower-pressure range $(5-16 \mathrm{GPa})$ and $0.4 \mathrm{GPa}$ 
in the upper range $(17-23 \mathrm{GPa})$ and thus typically within the general experimental uncertainty. This indicates that these heating cycles at a fixed load can be regarded as isobaric processes. The present experimental data on the phase diagram of $\mathrm{Pr}$ are represented in Fig. 1 together with earlier results. ${ }^{7,9,12-14,25}$ The data ${ }^{14}$ for the $\operatorname{Pr}$ (III)$\operatorname{Pr}(\mathrm{IV})$ phase boundary below $300 \mathrm{~K}$ were shifted by $10 \%$ to higher pressures to take into account the later revision ${ }^{9}$ of the corresponding pressure determinations.

The $\operatorname{Pr}(\mathrm{II})-\operatorname{Pr}(\mathrm{III})$ phase boundary was determined primarily in isobaric temperature scans, and Fig. 2 shows typical EDXD spectra at $12.3 \mathrm{GPa}$, for $\operatorname{Pr}(\mathrm{III})$ at $300 \mathrm{~K}$ and for $\operatorname{Pr}(\mathrm{II})$ at $403 \mathrm{~K}$, respectively, with an indexing of the superlattice diffraction lines by half-integer numbers. It can be seen that the intensities of the superlattice diffraction lines decrease with increasing temperature, and thus the temperature at which these diffraction lines disappear was taken as the temperature of the transition between $\operatorname{Pr}($ II) and $\operatorname{Pr}($ III). The question of whether this phase transition is truly second order, however, could not be resolved with this technique. ${ }^{16}$ Since the present EDXD setup, optimized for stronger intensities, does not resolve the $\left(\frac{3}{2}, \frac{1}{2}, \frac{1}{2}\right)$ peak from its much stronger (111) neighbor and leads also to an overlap of the $\left(\frac{3}{2}, \frac{3}{2}, \frac{1}{2}\right)$ peak with the fluorescence $K \alpha$ peak at higher pressures, the transition temperatures were determined by fitting two Gaussian profiles and a linear background to the (220)-

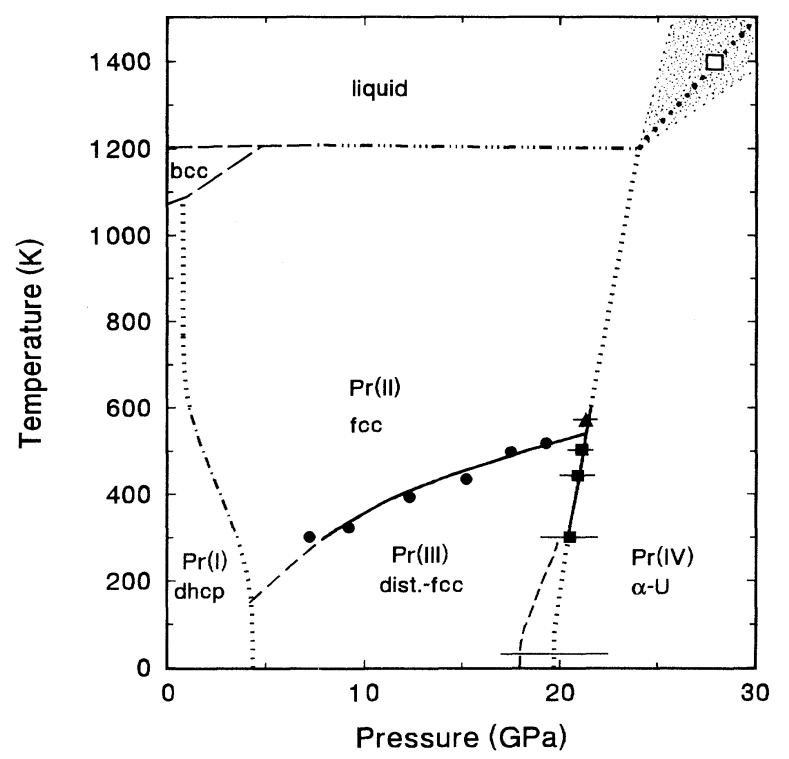

FIG. 1. Pressure-temperature phase diagram of Pr. The present data are represented by solid symbols with the solid lines as guides to eye. The dot-dashed line is from Ref. 7. The long-dashed lines are from Refs. 12 and 13. The tripledotted-dashed line reproduces the calculated melting line from shock wave data (Ref. 25). The short-dashed lines are from Heinrichs and Wittig (Ref. 14) and Wittig (Ref. 9) with appropriate corrections discussed in the text. A Hugoniot melting point from the shock wave data (Ref. 25) is represented by the open square and dotted lines represent extrapolations and estimates as discussed in the text.

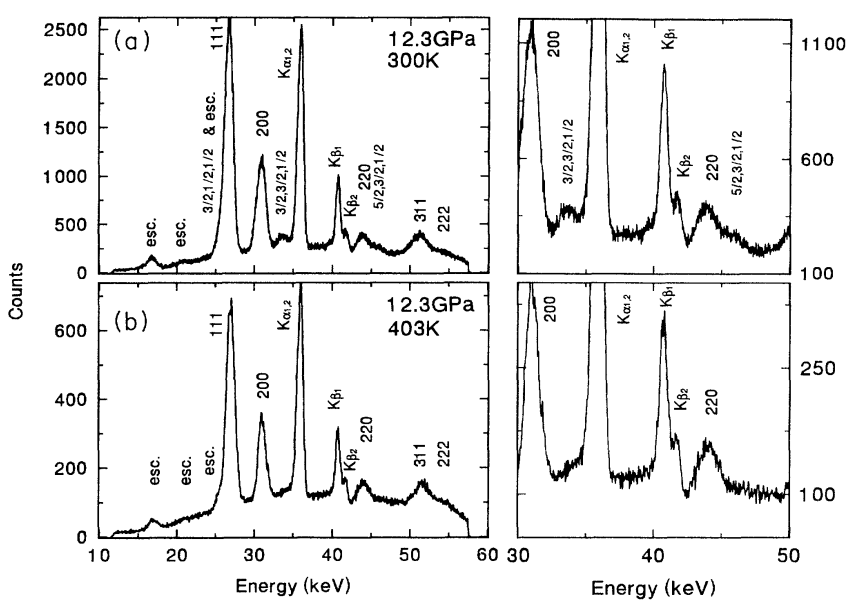

FIG. 2. Diffraction spectra for (a) $\operatorname{Pr}$ (III) at $300 \mathrm{~K}$ and (b) $\operatorname{Pr}(\mathrm{II})$ at $403 \mathrm{~K}$ at $12.3 \mathrm{GPa}$. The enlarged sections of these spectra on the right side illustrate the intensity variation of the superlattice reflections.

$\left(\frac{5}{2}, \frac{3}{2}, \frac{1}{2}\right)$ pair, keeping the full width at half maximum (FWHM) of the Gaussian profiles fixed to their 300-K values. Figure 3 shows a typical variation of the intensity of these two lines with temperature, where both intensities were normalized with respect to the intensity of the $K \beta$ doublet. From the decrease in the intensity of the $\left(\frac{5}{2}, \frac{3}{2}, \frac{1}{2}\right)$ peak, one finds at this pressure of $15.2 \mathrm{GPa}$ a transition temperature of $435(15) \mathrm{K}$. The same method was used for determining the transition temperatures at other pressures, and the results are shown in Fig. 1 by solid circles.

The phase boundary between $\operatorname{Pr}($ III) and $\operatorname{Pr}($ IV) was determined both in isothermal and isobaric scans. Figure 4 shows typical diffraction spectra for the $\operatorname{Pr}($ IV $) \rightarrow \operatorname{Pr}($ III $)$ transformation under isothermal decompression. Figure 5 presents diffraction spectra for
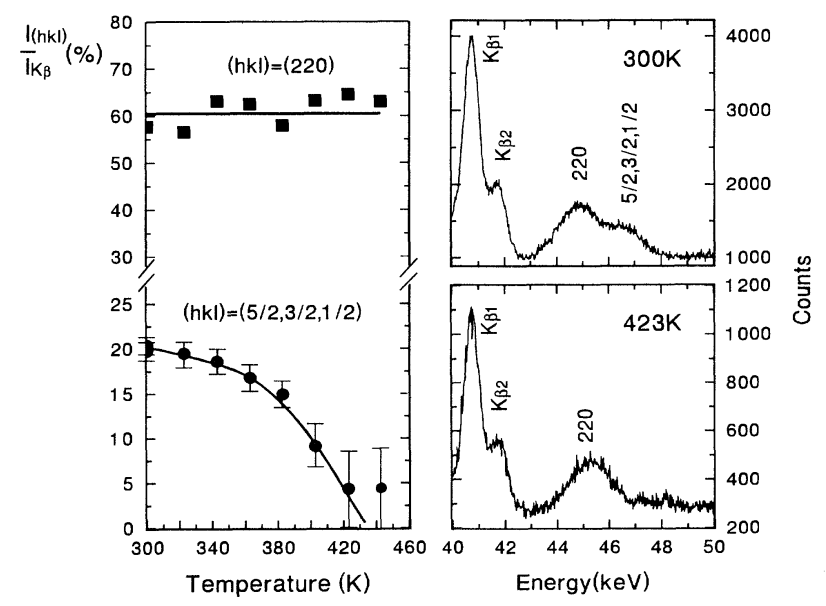

FIG. 3. Typical variation of scaled intensities with temperature at $15.2 \mathrm{GPa}$. The two spectra at this pressure illustrate the intensity variation of the $\left(\frac{5}{2}, \frac{3}{2}, \frac{1}{2}\right)$ peak. 
the transformation from $\operatorname{Pr}$ (III) (metastable at $300 \mathrm{~K}$ and $21.9 \mathrm{GPa})$ to $\operatorname{Pr}(\mathrm{IV})$ at an isobaric $21.9-\mathrm{GPa}$ scan from 300 to $325 \mathrm{~K}$. In contrast to the apparently continuous $\operatorname{Pr}($ II)-Pr(III) phase transition, considerable hysteresis was observed in the $\operatorname{Pr}($ III $) \rightarrow \operatorname{Pr}($ IV $)$ transformation, where the equilibrium phase transition pressures were taken therefore as the midpoints between the forward and backward transformations at constant temperature $T$. These data are illustrated in Fig. 1 by solid squares with horizontal lines indicating the width of the hysteresis loop.

One data point for the $\operatorname{Pr}(\mathrm{II})-\operatorname{Pr}(\mathrm{IV})$ transition measured at $573 \mathrm{~K}$ is also shown in Fig. 1 by a solid triangle. An EDXD spectrum for $\operatorname{Pr}(\mathrm{II})$ around this data point is shown in Fig. 6. The extrapolation of the II-III boundary toward the stability area of $\operatorname{Pr}(\mathrm{IV})$ locates the triple point at $21(1) \mathrm{GPa}$ and $537(15) \mathrm{K}$. Since the phase transitions $\operatorname{Pr}($ III)-Pr(IV) and $\operatorname{Pr}($ II) $-\operatorname{Pr}($ IV) are strongly first order and the $\operatorname{Pr}($ II) $)-\operatorname{Pr}($ III) is apparently of second order, it follows from the Clausius-Clapeyron equation that both the II-IV and III-IV transition lines must have (apparently) the same slopes at the triple point; i.e., there is no kink. The same reasoning applies to the I-II-III triple point.

In addition to the earlier estimates ${ }^{25}$ of the melting line for $\operatorname{Pr}(\mathrm{II})$, one can use also for $\operatorname{Pr}(\mathrm{IV})$ the Lindemann melting relation ${ }^{26}$ with the Debye-Grüneisen parameter $\gamma$ and its estimate from isothermal compression data with $V\left(\partial^{2} P / \partial V^{2}\right) /(\partial P / \partial V)=K_{T}^{\prime}$ and the DugdaleMacDonald relation $\gamma=\left(K_{T}^{\prime}-1\right) / 2$ to derive the relation

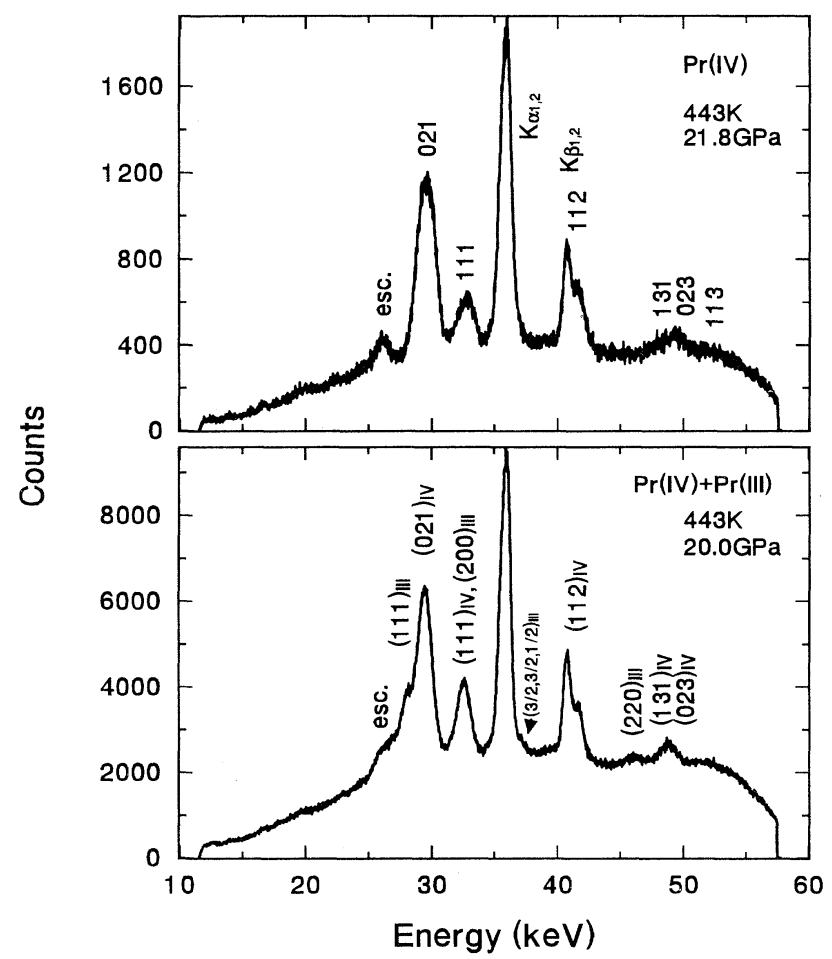

FIG. 4. Diffraction spectra for the isothermal $\operatorname{Pr}(\mathrm{IV}) \rightarrow \operatorname{Pr}(\mathrm{III})$ transformation at $443 \mathrm{~K}$ taken on decompression from 21.8 to $20.0 \mathrm{GPa}$. Acquisition times are $3 \mathrm{~h}$ for the first and $14 \mathrm{~h}$ for the second spectrum, respectively.

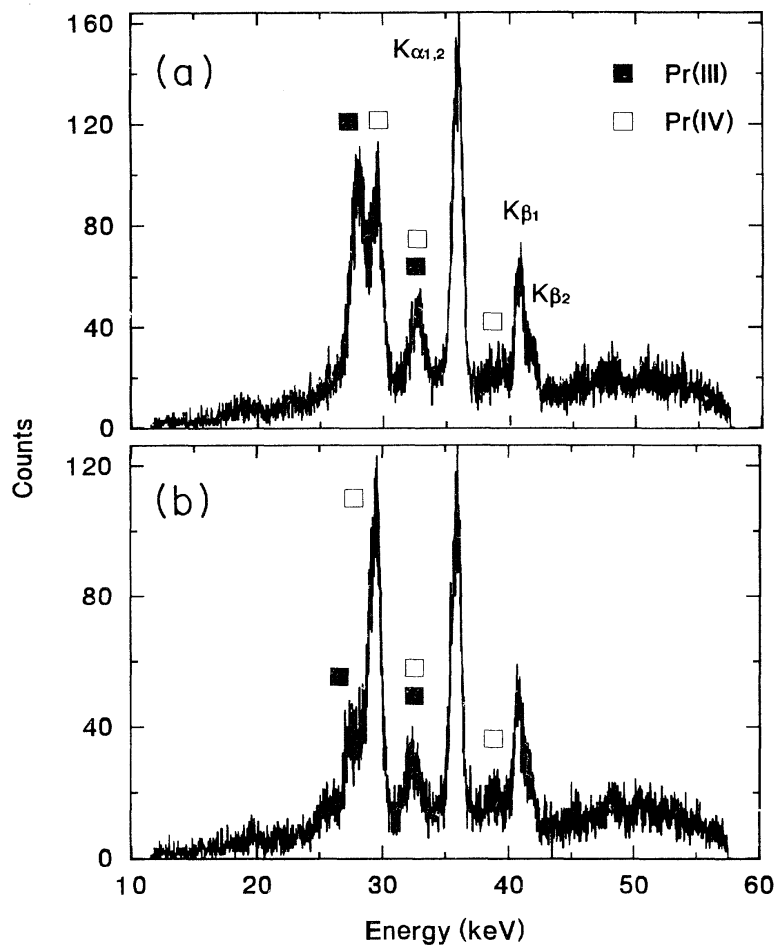

FIG. 5. Diffraction spectra in an isobaric scan at $21.9 \mathrm{GPa}$ for the $\operatorname{Pr}(\mathrm{III}) \rightarrow \operatorname{Pr}(\mathrm{IV})$ transformation at $325 \mathrm{~K}$ with typical acquisition times of $100 \mathrm{~s}$. (a) and (b) are the spectrum for this transition during first 100 and $300 \mathrm{~s}$, respectively.

$d T_{m} / d P=T_{m}\left(2 \gamma-\frac{2}{3}\right) / K_{T}=T_{m}\left(K_{T}^{\prime}-\frac{5}{3}\right) / K_{T}$. This relation reproduces the very small slope for the melting line of $\operatorname{Pr}(\mathrm{II})$, when one inserts the respective value ${ }^{6}$ of $K_{T}^{\prime}=2$, and one calculates a significantly larger slope for the melting curve of $\operatorname{Pr}(\mathrm{IV})$ when the corresponding values ${ }^{6}$ of $K_{T}^{\prime}=5$ and $K_{T}=120 \mathrm{GPa}$ are taken into account. Since the value for $K_{T}$ at melting may be somewhat

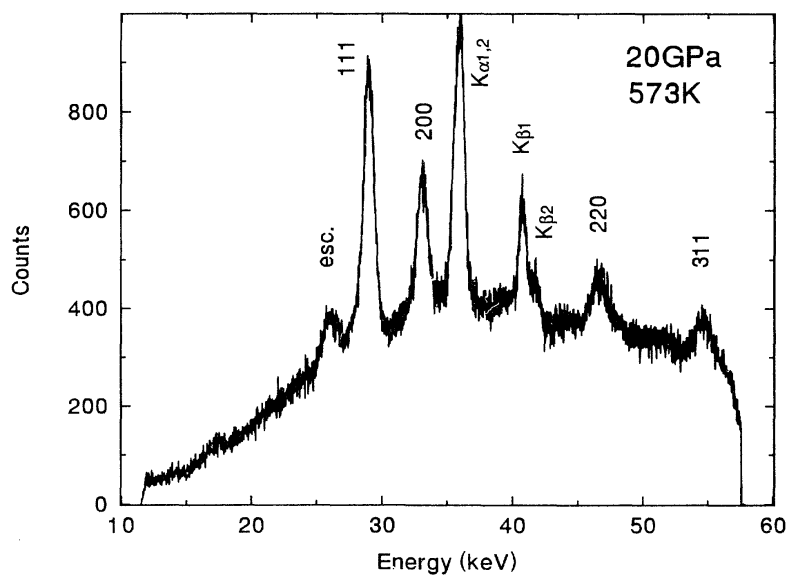

FIG. 6. Diffraction spectrum at $573 \mathrm{~K}$ and $20 \mathrm{GPa}$ with the escape peak from the $K \alpha$ lines denoted by "esc" and indexing of the diffraction lines according to the fcc structure of $\operatorname{Pr}$ (II). 
lower, this slope represents a lower limit for the melting line of $\operatorname{Pr}(\mathrm{IV})$. On the other hand, one can also use the Clausius-Clapeyron relation $d T / d P=\Delta V / \Delta S$ for both sections of the melting curve. The measurements show that value of $\Delta V / V$ for the $\operatorname{Pr}($ III $) \rightarrow \operatorname{Pr}($ IV $)$ phase transition decreases from $13 \%$ at ambient temperature to $7 \%$ at $573 \mathrm{~K}$. Therefore $\Delta V / V=13 \%$ gives an upper limit of the volume change at high temperatures. Since $\Delta S_{\text {melting }}$ for both sections of the melting curve can be regarded as being almost constant $\Delta S_{\text {melting }} \sim R$ and large compared with $\Delta S_{\text {II-IV }}$ for the II-IV transition near the triple point, the Clausius-Clapeyron relation results in an increase in the slope of the melting curve at the triple point from almost 0 to about $170 \mathrm{~K} / \mathrm{GPa}$, which is considered as an upper limit for the slope of this melting line. Shock wave studies $^{25}$ on Ln metals located the melting point on the Hugoniot curve for $\mathrm{Pr}$ around $28 \mathrm{GPa}$ and $1400 \mathrm{~K}$ as shown in Fig. 1 by an open square, and it was noted ${ }^{25}$ that this value for the melting temperature was much higher than their calculated melting line shown as the triple-dotted-dashed line in Fig. 1. The present study offers an explanation for this apparent discrepancy by the observation that the Hugoniot curve may intercept through the phase $\operatorname{Pr}(\mathrm{IV})$ with its much steeper melting curve. The connection between the triple point and the Hugoniot melting point is shown in Fig. 1 by a dotted line, which represents the present best data for melting of $\operatorname{Pr}(\mathrm{IV})$ right in the middle between the estimated lower and upper limits.

In summary, one observes a topology of the $P$ - $T$ phase diagram for $\mathrm{Pr}$, which shows a strong similarity to the phase diagram ${ }^{26}$ of $\mathrm{Ce}$ in its region of the $4 f$ delocalization.

The authors would like to thank Professor K. A. Gschneidner, Jr. for providing the sample material and W. Sievers and W. Bröckling for technical assistance. Y.C.Z. is indebted to Alexander von Humboldt-Stiftung for financial support.
*Permanent address: Beijing Synchrotron Radiation Facility, Institute of High Energy Physics, Beijing 100039, China.

${ }^{1}$ A. Jayaraman and R. C. Sherwood, Phys. Rev. Lett. 12, 22 (1964).

${ }^{2}$ D. B. McWhan and W. L. Bond, Rev. Sci. Instrum. 35, 626 (1964).

${ }^{3}$ G. J. Piermarini and C. E. Weir, Science 144, 69 (1964).

${ }^{4}$ K. A. Gschneidner, Jr. and F. W. Calderwood, in Handbook on the Physics and Chemistry of Rare Earths, edited by K. A. Gschneidner, Jr. and L. Eyring (North-Holland, Amsterdam, 1986), Vol. 8, p. 1.

${ }^{5}$ U. Benedict, W. A. Grosshans, and W. B. Holzapfel, Physica 144B, 14 (1986).

${ }^{6}$ W. A. Grosshans, Ph.D. thesis, University of Paderborn, 1987; W. A. Grosshans and W. B. Holzapfel, Phys. Rev. B 45, 5171 (1992).

${ }^{7}$ T. Krüger, B. Merkau, W. A. Grosshans, and W. B. Holzapfel, High Press. Res. 2, 193 (1990).

${ }^{8}$ U. Benedict and W. B. Holzapfel, in Handbook on the Physics and Chemistry of Rare Earths, edited by K. A. Gschneidner, Jr., L. Eyring, G. H. Lander, and G. R. Choppin (NorthHolland, Amsterdam, 1993), Vol. 17, p. 245.

${ }^{9} \mathrm{~J}$. Wittig, in Physics of Solids under High Pressure, edited by J. S. Schilling and R. N. Shelton (North-Holland, Amsterdam, 1981), p. 283.

${ }^{10}$ H. K. Mao, R. M. Hazen, P. M. Bell, and J. Wittig, J. Appl. Phys. 52, 4572 (1981).

${ }^{11}$ G. S. Smith and J. Akella, Phys. Lett. 105A, 132 (1984).

${ }^{12}$ A. J. Jayaraman, Phys. Rev. 139, A690 (1965).

${ }^{13}$ D. R. Stephens, Phys. Chem. Solids 26, 943 (1965).

${ }^{14}$ M. Heinrichs and J. Wittig, J. Phys. (Paris) Colloq. 39, C161072 (1978)

${ }^{15}$ N. Hamaya, Y. Sakamoto, H. Fujihisa, Y. Fujii, K. Takemu- ra, T. Kikegawa, and O. Shimomura, J. Phys. Condens. Matter 5, L369 (1993).

${ }^{16}$ F. Porsch and W. B. Holzapfel, Phys. Rev. B 50, 16212 (1994).

${ }^{17}$ K. Syassen and W. B. Holzapfel, Europhys. Conf. Abstr. 1A, 75 (1975)

${ }^{18}$ W. B. Holzapfel, in High Pressure Chemistry, edited by $\mathrm{H}$. Kelm (Reidel, Boston, 1978), p. 177.

${ }^{19}$ W. B. Holzapfel and W. May, in High-Pressure Research in Geophysics, edited by S. Akimoto and M. H. Manghnani (Center for Academic Publication Japan, Tokyo, and Reidel, Dordrecht, 1982), p. 73.

${ }^{20}$ H. K. Mao, P. Bell, J. W. Shaner, and D. J. Steinberg, J. Appl. Phys. 49, 3276 (1978).

${ }^{21}$ J. C. Jamieson, J. N. Fritz, and M. H. Manghnani, in Ref. 19, p. 27.

${ }^{22}$ L. C. Ming, M. H. Manghnani, J. Balogh, S. B. Qadri, E. F. Skelton, and J. C. Jamieson, J. Appl. Phys. 54, 4390 (1983).

${ }^{23}$ T. E. Scott, in Handbook on the Physics and Chemistry of Rare Earths, edited by K. A. Gschneidner, Jr. and L. Eyring (North-Holland, Amsterdam, 1978), Vol. 1, Chap. 8, p. 591.

${ }^{24}$ The Slater Grüneisen parameter $\gamma_{S, 0}=K_{0}^{\prime} / 2-\frac{1}{6}=0.83$ was derived from the value of the isothermal bulk modulus $K_{0}^{\prime}=2$ given by Ref. 8 . The thermal Grüneisen parameter $\gamma_{\text {th, } 0}=0.34$ was given in W. J. Cater, J. N. Fritz, S. P. Marsh, and R. G. McQueen, J. Phys. Chem. Solids 36, 741 (1975). The Dugdale-MacDonald's $\gamma_{\mathrm{DM}, 0}=0.45$ was given in W. $\mathrm{H}$. Gust and E. B. Royce, Phys. Rev. B 8, 3595 (1973). These values of $\gamma_{\mathrm{th}, 0}, \gamma_{S, 0}$, and $\gamma_{\mathrm{DM}, 0}$ all result in small thermal corrections in pressure.

${ }^{25}$ W. J. Cater, J. N. Fritz, S. P. Marsh, and R. G. McQueen, J. Phys. Chem. Solids 36, 741 (1975).

${ }^{26} \mathrm{D}$. A. Young, Phase Diagrams of the Elements (University of California Press, Berkeley, 1990). 